\title{
Particle-Hole Symmetry of Charge Excitation Spectra in the Paramagnetic Phase of the Hubbard Model
}

\author{
Vu Hung Dao, RaYmond Frésard* \\ Normandie University, ENSICAEN, UNICAEN, CNRS, CRISMAT, 14000 Caen, France
}

\begin{abstract}
The Kotliar and Ruckenstein slave-boson representation of the Hubbard model allows to obtain an approximation of the charge dynamical response function resulting from the Gaussian fluctuations around the paramagnetic saddle-point in analytical form. Numerical evaluation in the thermodynamical limit yields charge excitation spectra consisting of a continuum, a gapless collective mode with anisotropic zero-sound velocity, and a correlation induced high-frequency mode at $\omega \approx U$. In this work we show that this analytical expression obeys the particle-hole symmetry of the model on any bipartite lattice with one atom in the unit cell. Other formal aspects of the approach are also addressed.
\end{abstract}

DOI: 10.12693/APhysPolA.133.336

PACS/topics: 71.10.Fd, 72.15.Nj, 71.30.+h

\section{Introduction}

Most peculiar properties of transition metal oxides that attract a lot of attention are believed to result from strong electronic correlations. A great variety of physical phenomena has been evidenced [1], with prominent examples being the striking metal-to-insulator transitions in vanadium sesquioxide [2-5], high- $\mathrm{T}_{\mathrm{c}}$ superconductivity in the cuprates [6, 7], non-Fermi liquid behavior in the vanadates [8], stripes in nickelates [9, 10] and cuprates $[11,12]$, or the colossal magnetoresistance observed in the manganites [13-16]. In addition, a whole series of promising materials for thermoelectric applications has been discovered [17-23].

The infancy of the microscopical modeling of strongly correlated systems dates to the early sixties with the introduction of the so-called one-band Hubbard Model [24-27],

$$
H=-t \sum_{\langle i, j\rangle, \sigma} f_{i \sigma}^{\dagger} f_{j \sigma}+U \sum_{i} n_{i \uparrow} n_{i \downarrow},
$$

that describes interacting fermions hopping on a lattice between nearest neighbor sites with amplitude $-t$. The screened Coulomb interaction is assumed local, and its strength on each site $i$ is given by $U$. It was later on extended by Oleś to multiband systems to better embrace the diversity of transition metal oxides [28]. A fundamental consequence of strong correlations was already recognized by Hubbard, who showed that they split the non-interacting tight-binding band and give rise to additional features in the excitation spectra including the upper Hubbard band (UHB) $[24,25]$. In fact, more recent investigations of the one-band Hubbard Model within dynamical mean-field theory revealed that its oneparticle excitation spectra generically consist of lower and upper Hubbard bands, together with a quasi-particle

*corresponding author; e-mail: Raymond.Fresard@ensicaen.fr peak $[29,30]$. These genuine interaction-driven features are reflected in two-particle excitation spectra. For instance, as shown in Fig. 1 the charge excitation spectra consist of a continuum, a zero-sound collective mode, and a high-frequency collective mode originating from the upper Hubbard band [31]. The latter escapes a description within perturbation theory.

$\operatorname{Im} \chi_{\mathrm{c}}$

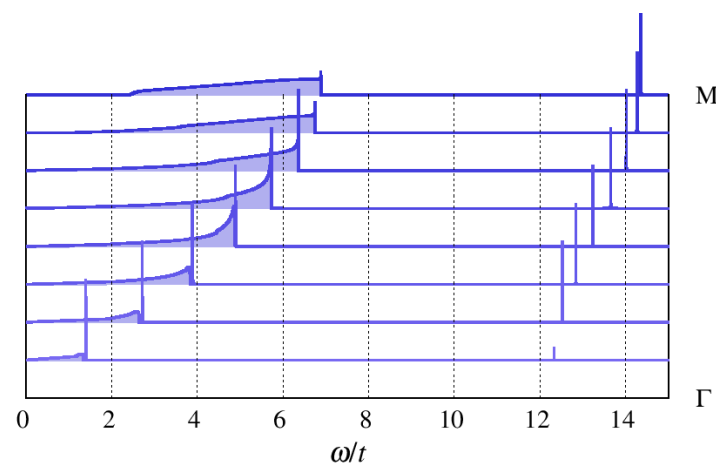

Fig. 1. Spectrum of the inelastic charge response function $\operatorname{Im} \chi_{c}$ at low temperature $T$ for different momenta along the path linking $\Gamma=(0,0)$ to $\mathrm{M}=(\pi, \pi)$, at coupling $U=10 t$ and doping from half-filling $\delta= \pm 0.5$. Note the peak of the zero-sound collective mode above the upper edge of the continuum, and the UHB mode peak at higher energy. Parameter: $T=t / 100$.

The Kotliar and Ruckenstein slave-boson representation of the Hubbard model [32] is a convenient tool to obtain these spectra at one-loop order in the paramagnetic phase [31]. Such a symmetry-breaking free calculation may be performed in the thermodynamical limit. It thus allows to resolve the full momentum dependence of the spectra. Since incommensurate magnetic instabilities are strongly suppressed with increasing temperature, neglecting magnetic instabilities does not severely constrain the parameter range where the calculation may be meaningfully performed. For instance, on the square lattice, they essentially disappear for $T \approx t / 6$ [31]. The Gutzwiller approximation, which entails the interaction 
driven Brinkman-Rice metal-to-insulator transition [33], is reproduced on the saddle-point level by the Kotliar and Ruckenstein slave-boson representations [32, 34].

The zero-temperature Brinkman-Rice metal-toinsulator transition is second order [33]. It turns first order at finite temperature as the latter may destabilize a poorly coherent Fermi Liquid [35, 36]. It is located in a coexistence region between a metallic and an insulating phase. Furthermore this first order transition extends to finite doping, where it is replaced by a transition from a good metal (with large quasi-particle residue) to a poor metal (with small quasi-particle residue). It extends up to a critical endpoint that depends on temperature [31, 37]. In contrast, a zero-temperature first order transition has been found in the two-band model in the vicinity of half-filling [38]. The role of the lattice geometry on the metal-to-insulator transition was also investigated [39]. In particular, a very good agreement on the location of the metal-to-insulator transition has been found with Quantum Monte Carlo simulations on the honeycomb lattice [35]. Further comparisons of groundstate energies to existing numerical solutions have also been carried out for the square lattice. Regarding groundstate energies, for instance for $U=4 t$, it could be shown that the slave-boson result is larger than its counterpart by less than $3 \%$ [40]. For larger values of $U$, the slave-boson groundstate energy exceeds the exact diagonalization data by less than $4 \%$ (7\%) for $U=8 t(20 t)$ and doping larger than $15 \%$. The discrepancy increases when the doping is lowered [41]. The saddle-point approximation is exact in the large degeneracy limit, and the Gaussian fluctuations provide $1 / N$ corrections [34]. Moreover it obeys a variational principle in the limit of large spatial dimensions where the Gutzwiller approximation becomes exact for the Gutzwiller wave function [42-44]. Let us finally emphasize that a quantitative agreement was established between the charge structure factors calculated from Gaussian fluctuations within the slave-boson approach and quantum Monte Carlo simulations [45].

Numerous valuable results have been obtained with Kotliar and Ruckenstein [32] and related slave-boson representations $[34,46]$. Special attention has been paid to anti-ferromagnetic [47], spiral [40,41, 48, 49], and striped [50-57] phases. In addition, the competition between the latter two has been addressed as well [57]. It has also been obtained that the spiral order continuously transforms to the ferromagnetic order in the large $U$ regime $(U \gtrsim 60 t)[49]$ so that its experimental realization is unlikely. Furthermore, in the two-band model on the square lattice, ferromagnetism was predicted as a possible groundstate in the doped Mott insulating regime only [38]. However, the ferromagnetic instability line could be brought down to the intermediate coupling regime when taking into account a ferromagnetic exchange coupling [58]. A sufficiently large next-nearest-neighbor hopping amplitude [59], as well as going to the fcc lattice [60], results in a similar effect. In addition, this formalism extended to the Hubbard model with inter-site Coulomb interaction has been applied to address the strongly inhomogeneous polaronic states observed in correlated heterostructures [61]. Most recently the possibility to enhance the capacitance by strong correlation effects in the metallic plates of a capacitor has been investigated within this approach [62].

Yet it needs to be verified that the approximate analytical expression used to calculate the charge excitation spectra complies with the symmetries of the model. Clearly, translational invariance and spin-rotational invariance are satisfied in a paramagnetic phase. However the less obvious particle-hole symmetry remains to be established. Furthermore, there is a certain degree of arbitrariness inherent to this representation as to how to perform the one-loop calculation: While the internal gauge symmetry group of the representation allows to simplify the problem, as the phase of three of the four slave-boson fields may be gauged away by promoting the Lagrange multipliers to time-dependent fields, there is no prescription to determine which one of them must remain a complex field. In this paper, we not only show that the charge excitation spectra computed in [31] are indeed particle-hole symmetric, but also that they do not depend on whether the selected complex slave-boson field describes doubly occupied sites or empty sites.

\section{Model and method}

In the spin-rotation invariant (SRI) Kotliar and Ruckenstein slave-boson representation [32,63] the Hubbard Hamiltonian is expressed with pseudo-fermion operators $f_{i \sigma}$ and auxiliary boson operators $e_{i}, p_{i \mu}, d_{i}$ (for atomic states with respectively zero, single and double occupancy) as

$$
H=-t \sum_{\langle i, j\rangle \sigma, \sigma^{\prime}, \sigma^{\prime \prime}} z_{i \sigma^{\prime \prime} \sigma}^{\dagger} f_{i \sigma}^{\dagger} f_{j \sigma^{\prime}} z_{j \sigma^{\prime} \sigma^{\prime \prime}}+U \sum_{i} d_{i}^{\dagger} d_{i} .
$$

In this form the on-site Coulomb interaction has the advantage to be bilinear in bosonic operators. The canonical operators $p_{i \mu}$ build a $2 \times 2$ matrix in spin space in order to preserve spin rotation symmetry [34, 46]. It is expanded into the identity matrix $\underline{\tau}^{0}$ and the Pauli matrices as $\underline{p}_{i}=\frac{1}{2} \sum_{\mu=0}^{3} p_{i \mu} \underline{\tau}^{\mu}$. The operator $\underline{z}_{i}$ takes into account the occupancy change that occurs during a hopping process. In the spin space it is also a matrix defined as

$$
\underline{z}_{i}=e_{i}^{\dagger} \underline{L}_{i} M_{i} \underline{R}_{i} \underline{p}_{i}+\underline{\tilde{r}}_{i}^{\dagger} \underline{R}_{i} M_{i} \underline{L}_{i} d_{i}
$$
with

$$
\begin{aligned}
& M_{i}=\left[1+e_{i}^{\dagger} e_{i}+\sum_{\mu=0}^{3} p_{i \mu}^{\dagger} p_{i \mu}+d_{i}^{\dagger} d_{i}\right]^{1 / 2}, \\
& \underline{L}_{i}=\left[\left(1-d_{i}^{\dagger} d_{i}\right) \underline{\tau}^{0}-2 \underline{p}_{i}^{\dagger} \underline{p}_{i}\right]^{-1 / 2}, \\
& \underline{R}_{i}=\left[\left(1-e_{i}^{\dagger} e_{i}\right) \underline{\tau}^{0}-2 \underline{\tilde{p}}_{i}^{\dagger} \tilde{p}_{i}\right]^{-1 / 2} \\
& \text { where } \underline{\tilde{p}}_{i}=\frac{1}{2}\left(p_{i 0} \underline{\tau}^{0}-\boldsymbol{p}_{i} \cdot \boldsymbol{\tau}\right) .
\end{aligned}
$$


The subspace of physical states in the augmented Fock space generated by the auxiliary fermion and boson operators is the intersection of the kernels of operators

$$
\begin{aligned}
\mathcal{A}_{i} & =e_{i}^{\dagger} e_{i}+\sum_{\mu=0}^{3} p_{i \mu}^{\dagger} p_{i \mu}+d_{i}^{\dagger} d_{i}-1, \\
\mathcal{B}_{i 0} & =\sum_{\mu=0}^{3} p_{i \mu}^{\dagger} p_{i \mu}+2 d_{i}^{\dagger} d_{i}-\sum_{\sigma} f_{i \sigma}^{\dagger} f_{i \sigma}, \\
\mathcal{B}_{i} & =p_{i 0}^{\dagger} \boldsymbol{p}_{i}+\boldsymbol{p}_{i}^{\dagger} p_{i 0}-\mathrm{i} \boldsymbol{p}_{i}^{\dagger} \times \boldsymbol{p}_{i}-\sum_{\sigma, \sigma^{\prime}} \boldsymbol{\tau}_{\sigma \sigma^{\prime}} f_{i \sigma^{\prime}}^{\dagger} f_{i \sigma},
\end{aligned}
$$

i.e. in this subspace $\mathcal{A}_{i}=0$ that is the constraint of one atomic state per site, and $\mathcal{B}_{i \mu}=0$ which equates the number of fermions to the number of $p$ and $d$ bosons.

Functional integration is used to calculate the partition function $[44,64]$ with the effective Lagrangian $\mathcal{L}=$ $\mathcal{L}^{\mathrm{B}}+\mathcal{L}^{\mathrm{F}}$. Here the purely bosonic part is

$$
\begin{aligned}
\mathcal{L}^{\mathrm{B}} & =\sum_{i}\left[e_{i}^{\dagger} \partial_{\tau} e_{i}+\sum_{\mu=0}^{3} p_{i \mu}^{\dagger} \partial_{\tau} p_{i \mu}+d_{i}^{\dagger}\left(\partial_{\tau}+U\right) d_{i}\right. \\
& \left.+\alpha_{i} \mathcal{A}_{i}+\sum_{\mu=0}^{3} \beta_{i \mu} \mathcal{B}_{i \mu}^{\mathrm{B}}\right]
\end{aligned}
$$

with $\mathcal{B}_{i \mu}^{\mathrm{B}}$ being the bosonic part of the operator $\mathcal{B}_{i \mu}$. After the integration of the fermion fields the mixed fermionboson part may be written as

$$
\begin{aligned}
& \mathcal{L}^{\mathrm{F}}=-\operatorname{tr}\left\{\operatorname { l n } \left[\left(\partial_{\tau}-\mu+\beta_{i 0}\right) \delta_{\sigma \sigma^{\prime}} \delta_{i j}+\boldsymbol{\beta}_{i} \cdot \boldsymbol{\tau}_{\sigma \sigma^{\prime}} \delta_{i j}\right.\right. \\
& \left.\left.\quad+t_{i j} \sum_{\sigma_{1}} z_{j \sigma \sigma_{1}}^{\dagger} z_{i \sigma_{1} \sigma^{\prime}}\right]\right\},
\end{aligned}
$$

with $\mu$ the chemical potential. The constraints that define the physical states are enforced with Lagrange multipliers $\alpha_{i}$ and $\beta_{i \mu}$. The problem may be simplified thanks to the internal gauge symmetry group of the representation. Indeed by promoting the Lagrange multipliers to time-dependent fields [34], the phases of $e$ and $p_{\mu}$ can be gauged away. This leaves us with radial slave-boson fields [65]. Their saddle-point values may be viewed as an approximation to their exact expectation values that are generically non-vanishing [66]. In fact, disproving earlier claims [67-69], the slave-boson field corresponding to double occupancy $d_{i}=d_{i}^{\prime}+\mathrm{i} d_{i}^{\prime \prime}$ however has to remain complex as emphasized by several authors [34, 70, 71]. Furthermore the dynamics of the, now, real $e_{i}$ and $p_{i \mu}$ fields drops out of $\mathcal{L}^{\mathrm{B}}$ due to the periodic boundary conditions on boson fields.

Within the approximation of Gaussian fluctuations, the action is expanded to second order in field fluctuations

$$
\begin{gathered}
\psi(k)=\left(\delta e(k), \delta d^{\prime}(k), \delta d^{\prime \prime}(k), \delta p_{0}(k), \delta \beta_{0}(k), \delta \alpha(k),\right. \\
\left.\delta p_{1}(k), \delta \beta_{1}(k), \delta p_{2}(k), \delta \beta_{2}(k), \delta p_{3}(k), \delta \beta_{3}(k)\right)
\end{gathered}
$$

around the paramagnetic saddle-point solution

$$
\psi_{\mathrm{MF}}=\left(e, d, 0, p_{0}, \beta_{0}, \alpha, 0,0,0,0,0,0\right)
$$

$$
\int \mathrm{d} \tau \mathcal{L}(\tau)=\mathcal{S}_{\mathrm{MF}}+\sum_{k, \mu, \nu} \psi_{\mu}(-k) S_{\mu \nu}(k) \psi_{\nu}(k)
$$

(the matrix $S$ is given in Appendix A of [31]). We have introduced the notation $k=\left(\boldsymbol{k}, \nu_{n}\right)$, with the Matsubara frequencies $\nu_{n}=2 \pi n T$, and $\sum_{k}=T \sum_{\nu_{n}} L^{-1} \sum_{k}$ with $L$ the number of lattice sites. The correlation functions of boson fields are then Gaussian integrals which can be obtained from the inverse of the fluctuation matrix $S$ as $\left\langle\psi_{\mu}(-k) \psi_{\nu}(k)\right\rangle=\frac{1}{2} S_{\mu \nu}^{-1}(k)$. Using the density fluctuation $\delta \mathcal{N}=\delta\left(d^{\dagger} d-e^{\dagger} e\right)$, the charge susceptibility is

$$
\begin{aligned}
& \chi_{c}(k)=\langle\mathrm{d} \mathcal{N}(-k) \mathrm{d} \mathcal{N}(k)\rangle \\
& \quad=2 e^{2} S_{1,1}^{-1}(k)-4 e d S_{1,2}^{-1}(k)+2 d^{2} S_{2,2}^{-1}(k) .
\end{aligned}
$$

Dynamical response functions are eventually evaluated within analytical continuation $\mathrm{i} \nu_{n} \rightarrow \omega+\mathrm{i} 0^{+}$.

\section{Symmetry of the saddle-point solution}

The Hubbard model possesses the particle-hole symmetry on a bipartite lattice. We show below that the symmetry is preserved at the saddle-point level in the specific case of the square lattice.

At first we present the general results of the paramagnetic solution, which do not presume any property of the lattice. At the saddle-point level, the boson values can be expressed with the hole doping from half-filling $\delta$ and the variable $x=e+d$ as

$$
e=\frac{x^{2}+\delta}{2 x}, \quad d=\frac{x^{2}-\delta}{2 x}, \quad p_{0}^{2}=1-\frac{x^{4}+\delta^{2}}{2 x^{2}} .
$$

The bare quasiparticle dispersion $t_{\boldsymbol{k}}$ is renormalized as

$$
E_{\boldsymbol{k}}=z_{0}^{2} t_{\boldsymbol{k}}-\mu_{\mathrm{eff}}
$$

with the factor

$$
z_{0}^{2}=\frac{2 p_{0}^{2}(e+d)^{2}}{1-\delta^{2}}
$$

and $\mu_{\text {eff }}$ the effective chemical potential.

The paramagnetic solution for fixed values of doping $\delta$ and coupling $U$ is found by determining the chemical potential $\mu_{\text {eff }}$ via the filling condition

$$
\frac{2}{L} \sum_{\boldsymbol{k}} n_{F}\left(E_{\boldsymbol{k}}\right)=1-\delta
$$

and the solution $x$ of the saddle-point equation

$$
\frac{\left(1-x^{2}\right) x^{4}}{x^{4}-\delta^{2}}=\frac{U}{U_{0}} .
$$

Here the coupling scale

$$
U_{0}=-8 \varepsilon_{0} /\left(1-\delta^{2}\right)
$$

has been introduced in terms of the semi-renormalized kinetic energy

$$
\varepsilon_{0}=\frac{2}{L} \sum_{\boldsymbol{k}} t_{\boldsymbol{k}} n_{F}\left(E_{\boldsymbol{k}}\right)
$$

and the Fermi function $n_{F}(\epsilon)=1 /(\exp (\epsilon / T)+1)$.

Solving the saddle-point equation (Eq. (16)) at halffilling yields $z_{0}^{2}=1-\left(U / U_{0}\right)^{2}$, in which case the effective mass of the quasiparticles diverges when $U$ reaches $U_{0}$. This is the Brinkman-Rice mechanism of the metal-toinsulator transition, as it also arises in the Gutzwiller approximation $[33,72,73]$. In order to establish the 
particle-hole symmetry of the saddle-point approximation, we show that the solution for the opposite doping is obtained by swapping the values of the empty and double occupancy, $e$ and $d$, and reversing the sign of the effective chemical potential $\mu_{\mathrm{eff}}$, while keeping unchanged $p_{0}$. Hence the saddle-point value $x$ and the renormalization factor $z_{0}$ are even functions of $\delta$. The boson expressions (12) comply with the transformation, so it remains to check that the latter (i) leaves invariant the saddlepoint equation and (ii) yields the filling condition for the opposite doping. This can be achieved using the parity of the quasiparticle density of state $N(-\epsilon)=N(\epsilon)$. Alternatively, the dispersion on the square lattice

$$
t_{\boldsymbol{k}}=-2 t\left(\cos k_{x}+\cos k_{y}\right)
$$

yields the property $t_{\boldsymbol{k}+\boldsymbol{Q}}=-t_{\boldsymbol{k}}$, with $\boldsymbol{Q}=(\pi, \pi)$, that will be used in the next section.

For (i) one can remark that both sides of Eq. (16) are even in $\delta$ since

$$
\begin{aligned}
\varepsilon_{0}(-\delta)=\frac{2}{L} \sum_{\boldsymbol{k}} t_{\boldsymbol{k}} n_{F}\left(z_{0}^{2} t_{\boldsymbol{k}}+\mu_{\mathrm{eff}}\right) & = \\
\frac{2}{L} \sum_{\boldsymbol{k}^{\prime}} t_{\boldsymbol{k}^{\prime}+\boldsymbol{Q}^{\prime}} n_{F}\left(z_{0}^{2} t_{\boldsymbol{k}^{\prime}+\boldsymbol{Q}}+\mu_{\mathrm{eff}}\right) & = \\
\frac{2}{L} \sum_{\boldsymbol{k}^{\prime}} t_{\boldsymbol{k}^{\prime}}\left[n_{F}\left(z_{0}^{2} t_{\boldsymbol{k}^{\prime}}-\mu_{\mathrm{eff}}\right)-1\right] & =\varepsilon_{0}(\delta)
\end{aligned}
$$

(the last equality results from the vanishing of $\sum_{k} t_{k}$ over the Brillouin zone) so the saddle-point equation is invariant. As for (ii), the density of the transformed solution

$$
\begin{aligned}
& \frac{2}{L} \sum_{\boldsymbol{k}} n_{F}\left(z_{0}^{2} t_{\boldsymbol{k}}+\mu_{\mathrm{eff}}\right)=\frac{2}{L} \sum_{\boldsymbol{k}^{\prime}} n_{F}\left(z_{0}^{2} t_{\boldsymbol{k}^{\prime}+\boldsymbol{Q}}+\mu_{\mathrm{eff}}\right)= \\
& \frac{2}{L} \sum_{\boldsymbol{k}^{\prime}}\left[1-n_{F}\left(z_{0}^{2} t_{\boldsymbol{k}^{\prime}}-\mu_{\mathrm{eff}}\right)\right]= \\
& 2-(1-\delta)=1+\delta
\end{aligned}
$$

indeed corresponds to the opposite doping.

\section{Symmetry of the quasiparticle response functions}

On the square lattice the quasiparticle response function is transformed under the reversal of the doping sign as

$$
\chi_{m}^{-}(k)=(-1)^{m} \chi_{m}^{+}(k) .
$$

Here we have introduced the notation for the generalized quasiparticle response functions at doping $\pm \delta$

$$
\chi_{m}^{ \pm}(k)=\frac{2}{L} \sum_{\boldsymbol{q}}\left(t_{\boldsymbol{q}+\boldsymbol{k}}+t_{\boldsymbol{q}}\right)^{m} \frac{n_{F}\left(E_{\boldsymbol{q}+\boldsymbol{k}}^{ \pm}\right)-n_{F}\left(E_{\overline{\boldsymbol{q}}}^{ \pm}\right)}{\omega-\left(E_{\boldsymbol{q}+\boldsymbol{k}}^{ \pm}-E_{\overline{\boldsymbol{q}}}^{ \pm}\right)}
$$

with $E_{\overline{\boldsymbol{q}}}^{ \pm}=z_{0}^{2} t_{\boldsymbol{q}} \mp \mu_{\mathrm{eff}}$. The relation between the expressions at opposite dopings can be derived by summing instead on $\boldsymbol{p}=-\boldsymbol{q}-\boldsymbol{k}+\boldsymbol{Q}$. This yields

$$
\begin{gathered}
\chi_{m}^{-}(k)=\frac{2}{L} \sum_{\boldsymbol{p}}\left(t_{-\mathbf{p}+\boldsymbol{Q}}+t_{-\boldsymbol{p}-\boldsymbol{k}+\boldsymbol{Q}}\right)^{m} \\
\times \frac{n_{F}\left(E_{-\boldsymbol{p}+\boldsymbol{Q}}^{-}\right)-n_{F}\left(E_{-\boldsymbol{p}-\boldsymbol{k}+\boldsymbol{Q}}^{-}\right)}{\omega-\left(E_{-\boldsymbol{p}+\boldsymbol{Q}}^{-}-E_{-\boldsymbol{p}-\boldsymbol{k}+\boldsymbol{Q}}^{-}\right)}
\end{gathered}
$$

$$
=\frac{2}{L} \sum_{\boldsymbol{p}}\left(-t_{\boldsymbol{p}}-t_{\boldsymbol{p}+\boldsymbol{k}}\right)^{m} \frac{n_{F}\left(-E_{\boldsymbol{p}}^{+}\right)-n_{F}\left(-E_{\boldsymbol{p}+\boldsymbol{k}}^{+}\right)}{\omega-\left(-E_{\boldsymbol{p}}^{+}+E_{\boldsymbol{p}+\boldsymbol{k}}^{+}\right)}
$$

wherein we have used the equalities $t_{-\boldsymbol{p}+\boldsymbol{Q}}=-t_{\boldsymbol{p}}$ and $E_{-\boldsymbol{p}+\boldsymbol{Q}}^{-}=-z_{0}^{2} t_{\boldsymbol{p}}+\mu_{\mathrm{eff}}=-E_{\boldsymbol{p}}^{+}$. Finally the sum can be written as

$$
\begin{gathered}
\chi_{m}^{-}(k)=(-1)^{m} \frac{2}{L} \sum_{\boldsymbol{p}}\left(t_{\boldsymbol{p}+\boldsymbol{k}}+t_{\boldsymbol{p}}\right)^{m} \\
\times \frac{n_{F}\left(E_{\boldsymbol{p}+\boldsymbol{k}}^{+}\right)-n_{F}\left(E_{\boldsymbol{p}}^{+}\right)}{\omega-\left(E_{\boldsymbol{p}+\boldsymbol{k}}^{+}-E_{\boldsymbol{p}}^{+}\right)}
\end{gathered}
$$

since $n_{F}(-\epsilon)=1-n_{F}(\epsilon)$.

En passant the above relation shows the particle-hole symmetry of the RPA charge response function

$$
\chi_{\mathrm{RPA}}(k)=\frac{\chi_{0}^{(0)}(k)}{1+\frac{U}{2} \chi_{0}^{(0)}(k)}
$$

where $\chi_{0}^{(0)}(k)$ is the charge response function of a Fermi gas, i.e. $\chi_{0}^{(0)}(k)=\left.\chi_{0}(k)\right|_{z_{0}=1}$.

\section{Symmetry of the slave-boson charge response}

The charge dynamical response function has been given in Ref. [31]. In order to demonstrate its particle-hole symmetry, we cast it into an expression which is explicitly invariant under the transformation $\{\delta \mapsto-\delta, e \mapsto d, d \mapsto$ $\left.e, \chi_{m}(k) \mapsto(-1)^{m} \chi_{m}(k)\right\}$ undergone when reversing the doping sign. After a lengthy but straightforward expansion, it can be written as

$$
\chi_{c}(k)=\frac{A(k)+B(k) \omega^{2}}{C(k)+D(k) \omega^{2}}
$$

where

$$
\begin{aligned}
& A(k)=\frac{2 p_{0}^{2} \varepsilon_{0}}{1-\delta^{2}}\left[\left(p_{0}^{2}\left(d^{2} S_{11}+e^{2} S_{22}\right)+4 e^{2} d^{2} S_{44}\right.\right. \\
& \left.\quad+2 e d p_{0}^{2} S_{12}-4 e d p_{0}\left(d S_{14}+e S_{24}\right)\right) \chi_{0}(k) \\
& \left.\quad+2\left(e \Delta_{1}-d \Delta_{2}\right)^{2}\right] \\
& B(k)=e d p_{0}^{2} \chi_{0}(k), \\
& \quad+4 e d S_{14} S_{24}-2 p_{0}\left(d S_{11} S_{24}+e S_{22} S_{14}\right) \\
& \left.\quad+\left(d^{2} S_{11}+e^{2} S_{22}-2 e d S_{12}\right) S_{44}\right) \frac{2 p_{0}^{2} \varepsilon_{0}}{1-\delta^{2}}\left[\left(p_{0}^{2} S_{11} S_{22}-\left(p_{0} S_{12}-d S_{14}-e S_{24}\right)^{2}\right.\right. \\
& \quad+S_{11} \Delta_{1}^{2}+S_{22} \Delta_{2}^{2}+S_{44} \Delta_{4}^{2}+2 S_{12} \Delta_{1} \Delta_{2} \\
& \left.\quad-2 S_{14} \Delta_{1} \Delta_{4}-2 S_{24} \Delta_{2} \Delta_{4}\right], \\
& D(k)=\frac{e d}{(e+d)^{2}}\left[\left(p_{0}^{2}\left(S_{11}+S_{22}\right)+(e-d)^{2} S_{44}\right.\right.
\end{aligned}
$$




$$
\begin{aligned}
& \left.-2 p_{0}^{2} S_{12}+2(e-d) p_{0}\left(S_{24}-S_{14}\right)\right) \frac{\chi_{0}(k)}{2} \\
& \left.+\left(\Delta_{1}+\Delta_{2}\right)^{2}\right] .
\end{aligned}
$$

Here the elements of the fluctuation matrix are

$$
\begin{aligned}
& S_{11}=-\varepsilon_{0} \frac{z_{0}}{e} \frac{\partial z}{\partial e}+s_{11}(k), \\
& S_{22}=-\varepsilon_{0} \frac{z_{0}}{d} \frac{\partial z}{\partial d^{\prime}}+s_{22}(k), \\
& S_{44}=-\varepsilon_{0} \frac{z_{0}}{p_{0}} \frac{\partial z}{\partial p_{0}}+s_{44}(k), \\
& S_{\mu \nu}=s_{\mu \nu}(k) \text { for } \mu, \nu=1,2,4 \text { with } \mu \neq \nu,
\end{aligned}
$$

where

$$
\begin{aligned}
& s_{\mu \nu}(k)=\varepsilon_{0} z_{0} \frac{\partial^{2} z}{\partial \psi_{\mu} \partial \psi_{\nu}} \\
& +\frac{\partial z}{\partial \psi_{\mu}} \frac{\partial z}{\partial \psi_{\nu}}\left[\varepsilon_{\boldsymbol{k}}-\frac{z_{0}^{2}}{2} \chi_{2}(k)\right],
\end{aligned}
$$

with

$$
\varepsilon_{\boldsymbol{k}}=\frac{2}{L} \sum_{\boldsymbol{q}} t_{\boldsymbol{q}+\boldsymbol{k}} n_{F}\left(E_{\boldsymbol{q}}\right)
$$

and

$$
\begin{aligned}
& \Delta_{1}=d p_{0}+\left(p_{0} \frac{\partial z}{\partial d^{\prime}}-d \frac{\partial z}{\partial p_{0}}\right) \frac{z_{0}}{2} \chi_{1}(k), \\
& \Delta_{2}=e p_{0}-\left(p_{0} \frac{\partial z}{\partial e}-e \frac{\partial z}{\partial p_{0}}\right) \frac{z_{0}}{2} \chi_{1}(k), \\
& \Delta_{4}=2 e d+\left(e \frac{\partial z}{\partial d^{\prime}}-d \frac{\partial z}{\partial e}\right) \frac{z_{0}}{2} \chi_{1}(k) .
\end{aligned}
$$

The expressions of the derivatives of $z$ may be gathered from Ref. [45, 64]. The first-order derivatives are

$$
\begin{gathered}
\frac{\partial z}{\partial e}=\sqrt{2} \eta p_{0}\left(1+\frac{2 x e}{1-\delta}\right), \\
\frac{\partial z}{\partial d^{\prime}}=\sqrt{2} \eta p_{0}\left(1+\frac{2 x d}{1+\delta}\right), \\
\frac{\partial z}{\partial p_{0}}=\sqrt{2} \eta x\left(1+\frac{2 p_{0}^{2}}{1-\delta^{2}}\right),
\end{gathered}
$$

with $\eta=1 / \sqrt{1-\delta^{2}}$. The second-order derivatives are

$$
\begin{aligned}
\frac{\partial^{2} z}{\partial e^{2}} & =\frac{2 \sqrt{2} \eta p_{0}}{1-\delta}\left(x+2 e+\frac{6 x e^{2}}{1-\delta}\right), \\
\frac{\partial^{2} z}{\partial d^{\prime 2}} & =\frac{2 \sqrt{2} \eta p_{0}}{1+\delta}\left(x+2 d+\frac{6 x d^{2}}{1+\delta}\right), \\
\frac{\partial^{2} z}{\partial p_{0}^{2}} & =2 \sqrt{2} \eta^{3} x p_{0}\left(3+\left(6 \eta^{2}-2\right) p_{0}^{2}\right), \\
\frac{\partial^{2} z}{\partial e \partial d^{\prime}} & =2 \sqrt{2} \eta p_{0}\left(\frac{e}{1-\delta}+\frac{d}{1+\delta}+2 \eta^{2} x e d\right), \\
\frac{\partial^{2} z}{\partial e \partial p_{0}} & =\sqrt{2} \eta\left(1+2 \eta^{2} p_{0}^{2}(1+x e)+\frac{2 x e}{1-\delta}+\frac{6 x e p_{0}^{2}}{(1-\delta)^{2}}\right),
\end{aligned}
$$

$$
\frac{\partial^{2} z}{\partial d^{\prime} \partial p_{0}}=\sqrt{2} \eta\left(1+2 \eta^{2} p_{0}^{2}(1+x d)+\frac{2 x d}{1+\delta}+\frac{6 x d p_{0}^{2}}{(1+\delta)^{2}}\right) .
$$

It should be emphasized that due to the symmetry of the saddle-point solution, i.e. $e(-\delta)=d(\delta)$ and $p_{0}(-\delta)=p_{0}(\delta)$, the values of the partial derivatives of $z$ by $e$ are interchanged with those by $d^{\prime}$ when reversing the sign of the doping, e.g. $\left(\partial^{2} z / \partial e \partial p_{0}\right)(-\delta)=$ $\left(\partial^{2} z / \partial d^{\prime} \partial p_{0}\right)(\delta)$. The values of the fluctuation matrix elements $S_{\mu \nu}$ with indices 1 and 2 are thus interchanged, e.g. $S_{11}(-\delta)=S_{22}(\delta)$ or $S_{14}(-\delta)=S_{24}(\delta)$. Furthermore one can check that $\Delta_{4}(-\delta)=\Delta_{4}(\delta)$ and $\Delta_{1}(-\delta)=\Delta_{2}(\delta)$. As a result, the coefficients (28), and then the response function, are actually invariant.

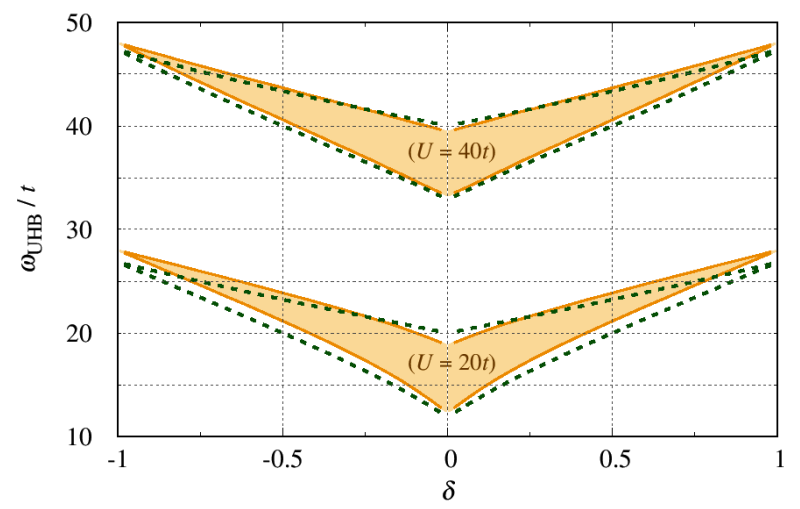

Fig. 2. Dispersion of the UHB mode following from Eq. (27) (shaded area) as a function of the doping for $U=20 t$ and 40t. The dashed boundaries are obtained from the strong-coupling approximation Eq. (35). Parameter: $T=t / 100$.

As an example we plotted the numerical evaluation of Eq. (27) in Fig. 1, where the characteristic features of the inelastic charge response function are clearly visible. While neither the continuum nor the zero-sound collective mode above the upper edge of the continuum could be brought into a simple analytical form, an approximate expression of the UHB pole for all momenta applicable in the strong coupling regime could be derived [31]. It reads

$$
\omega_{\mathrm{UHB}}(\boldsymbol{k}) \approx U \sqrt{1-\frac{U_{0}}{2 U}\left(1-3|\delta|+(1-|\delta|) \frac{\varepsilon_{\boldsymbol{k}}}{\varepsilon_{0}}\right)} .
$$

In Fig. 2 we compare the evaluation of Eq. (27) with the approximation Eq. (35) for $U=20 t$ and $40 t$. The smallest energy of the UHB mode peak is obtained for $\boldsymbol{k}=$ $(0,0)$, and the highest one for $\boldsymbol{k}=(\pi, \pi)$. The dispersion of this mode is approximately given by $-2(1-|\delta|) \varepsilon_{\boldsymbol{k}}$, and is accordingly largest at half-filling and vanishingly small in the limit of empty/full system. Furthermore, the difference between the approximation Eq. (35) and Eq. (27) tends to vanish already for $U=40 t$. 
Let us finally mention an alternative derivation of Eq. (27). We recall that there is some arbitrariness to its derivation as we chose here to gauge away the phase of the $e$-boson (on top of the one of the $p_{\mu}$-bosons). Alternatively one may chose to gauge away the phase of the $d$-boson while keeping the $e$-boson as a complex field. This obviously leads to a $S$-matrix that differs from the one derived in [31] and used here. Yet, tedious work shows that $\chi_{c}(k)$ obtained this way is nevertheless given by Eq. (27), thereby overcoming the above mentioned arbitrariness and putting Eq. (27) on a firmer ground.

\section{Conclusion}

Summarizing, the particle-hole symmetry of the charge response function obtained for the Hubbard model using various approximations has been considered. It has first been established that this symmetry is obeyed in the random phase approximation for a bipartite lattice such as the square lattice that we explicitly addressed. We then considered the expression of $\chi_{c}$ resulting from the SRI Kotliar and Ruckenstein slave boson representation calculated to one-loop order again on the square lattice. In this case we succeeded to cast its rather involved expression into a form that is manifestly particlehole symmetric. The latter also applies to the Kotliar and Ruckenstein representation. Our arguments can easily be extended to other bipartite lattices with one atom in the unit cell, such as the simple cubic one.

\section{Acknowledgments}

The authors acknowledge the financial support of the French Agence Nationale de la Recherche (ANR), through the program Investissements d'Avenir (ANR10-LABX-09-01), LabEx EMC3, the Région BasseNormandie, the Région Normandie, and the Ministère de la Recherche.

\section{References}

[1] M. Imada, A. Fujimori, Y. Tokura, Rev. Mod. Phys. 70, 1039 (1998).

[2] D. B. McWhan, A. Menth, J. P. Remeika, W. F. Brinkman, T. M. Rice, Phys. Rev. B 7, 1920 (1973).

[3] K. Held, G. Keller, V. Eyert, D. Vollhardt, V. I. Anisimiov, Phys. Rev. Lett. 86, 5345 (2001).

[4] P. Limelette, A. Georges, D. Jérome, P. Wzietek, P. Metcalf, J. M. Honig, Science 302, 89 (2003).

[5] C. Grygiel, Ch. Simon, B. Mercey, W. Prellier, R. Frésard, P. Limelette, Appl. Phys. Lett. 91, 262103 (2007).

[6] J. G. Bednorz, K. A. Müller, Z. Phys. B: Condens. Matter 64, 189 (1986).

[7] A. Schilling, M. Cantoni, J. D. Guo, H. R. Ott, Nature (London) 363, 56 (1993).

[8] F. Inaba, T. Arima, T. Ishikawa, T. Katsufuji, Y. Tokura, Phys. Rev. B 52, R2221(R) (1995).

[9] V. Sachan, D. J. Buttrey, J. M. Tranquada, J. E. Lorenzo, G. Shirane, Phys. Rev. B 51, R12742 (1995).
[10] R. Zhong, B. L. Winn, G. Gu, D. Reznik, J. M. Tranquada, Phys. Rev. Lett. 118, 177601 (2017).

[11] J. M. Tranquada, B. J. Sternlieb, J. D. Axe, Y. Nakamura, S. Uchida, Nature (London) 375, 561 (1995).

[12] Z. Guguchia, B. Roessli, R. Khasanov, A. Amato, E. Pomjakushina, K. Conder, Y.-J. Uemura, J.-M. Tranquada, H. Keller, A. Shengelaya, Phys. Rev. Lett. 119, 087002 (2017).

[13] R. von Helmolt, J. Wecker, B. Holzapfel, L. Schultz, K. Samwer, Phys. Rev. Lett. 71, 2331 (1993).

[14] Y. Tomioka, A. Asamitsu, Y. Moritomo, H. Kuwahara, Y. Tokura, Phys. Rev. Lett. 74, 5108 (1995).

[15] B. Raveau, A. Maignan, V. Caignaert, J. Solid State Chem. 117, 424 (1995).

[16] A. Maignan, Ch. Simon, V. Caignaert, B. Raveau, Solid State Commun. 96, 623 (1995).

[17] I. Terasaki, Y. Sasago, K. Uchinokura, Phys. Rev. B 56, R12685 (1997).

[18] A. C. Masset, C. Michel, A. Maignan, M. Hervieu, O. Toulemonde, F. Studer, B. Raveau, Phys. Rev. B 62, $166(2000)$.

[19] I. Matsubara, R. Funahashi, T. Takeuchi, S. Sodeoka, T. Shimizu, K. Ueno, Appl. Phys. Lett. 78, 3627 (2001); I. Matsubara, R. Funahashi, T. Takeuchi, S. Sodeoka, T. Shimizu, K. Ueno, Appl. Phys. Lett. 78, 3627 (2001).

[20] M. Miclau, J. Hejtmanek, R. Retoux, K. Knizek, Z. Jirak, R. Frésard, A. Maignan, S. Hébert, M. Hervieu, C. Martin, Chem. Matter. 19, 4243 (2007).

[21] H. Ohta et al, Nat. Mater. 6, 129 (2007).

[22] A. Maignan, V. Eyert, C. Martin, S. Kremer, R. Frésard, D. Pelloquin, Phys. Rev. B $\mathbf{8 0}$, 115103. (2009).

[23] N. Wang, H. J. Chen, H. C. He, W. Norimatsu, M. Kusunoki, K. Koumoto, Sci. Rep. 3, 3449 (2013).

[24] J. Hubbard, Proc. Roy. Soc. London A 276, 238 (1963).

[25] J. Hubbard, Proc. Roy. Soc. London A 281, 401 (1964).

[26] J. Kanamori, Prog. Theor. Phys. 30, 275 (1963).

[27] M. C. Gutzwiller, Phys. Rev. Lett. 10, 159 (1963).

[28] A. M. Oleś, Phys. Rev. B 28, 327 (1983).

[29] A. Georges, G. Kotliar, W. Krauth, M. J. Rozenberg, Rev. Mod. Phys. 68, 13 (1996).

[30] R. Bulla, T. A. Costi, D. Vollhardt, Phys. Rev. B 64 , 045103 (2001).

[31] V. H. Dao, R. Frésard, Phys. Rev. B 95, 165127 (2017).

[32] G. Kotliar, A. E. Ruckenstein, Phys. Rev. Lett. 57, 1362 (1986).

[33] W. F. Brinkman, T. M. Rice, Phys. Rev. B 2, 4302 (1970).

[34] R. Frésard, P. Wölfle, Int. J. of Mod. Phys. B 6, 685 (1992); ibid. 6, 3087 (1992). 
[35] R. Frésard, K. Doll, Proceedings of the NATO ARW, The Hubbard Model: Its Physics, Mathematical Physics, Eds. D. Baeriswyl, D. K. Campbell, J. M. P. Carmelo, F. Guinea, E. Louis, San Sebastian (1993), Plenum Press, 1995, p. 385.

[36] R. Frésard, G. Kotliar, Phys. Rev. B 56, 12909 (1997).

[37] A. Mezio, R. H. McKenzie, Phys. Rev. B 96, 035121 (2017).

[38] R. Frésard, M. Lamboley, J. Low Temp. Phys. 126, 1091 (2002).

[39] G. Kotliar, E. Lange, M. J. Rozenberg, Phys. Rev. Lett. 84, 5180 (2000).

[40] R. Frésard, M. Dzierzawa, P. Wölfle, Europhys. Lett. 15, 325 (1991).

[41] R. Frésard, P. Wölfle, J. Phys.: Condens. Matter 4, 3625 (1992)

[42] W. Metzner, D. Vollhardt, Phys. Rev. Lett. 62, 324 (1989).

[43] W. Metzner, D. Vollhardt, Phys. Rev. B 37, 7382 (1988).

[44] W. Metzner, Z. Phys. B 77, 253 (1989)

[45] W. Zimmermann, R. Frésard, P. Wölfle, Phys. Rev. B 56, 10097 (1997).

[46] T. C. Li, P. Wölfle, P. J. Hirschfeld, Phys. Rev. B 40, 6817 (1989)

[47] L. Lilly, A. Muramatsu, W. Hanke, Phys. Rev. Lett. 65, 1379 (1990)

[48] P. A. Igoshev, M. A. Timirgazin, A. K. Arzhnikov, V. Y. Irkhin, JETP Lett. 98, 150 (2013).

[49] B. Möller, K. Doll, R. Frésard, J. Phys.: Condens. Matter 5, 4847 (1993).

[50] G. Seibold, E. Sigmund, V. Hizhnyakov, Phys. Rev. B 57, 6937 (1998).

[51] M. Fleck, A. I. Lichtenstein, A. M. Oleś, Phys. Rev. B 64, 134528 (2001).

[52] J. Lorenzana, G. Seibold, Phys. Rev. Lett. 89, 136401 (2002).

[53] J. Lorenzana, G. Seibold, Phys. Rev. Lett. 90, 066404 (2003).

[54] J. Lorenzana, G. Seibold, Phys. Rev. Lett. 94, 107006 (2005).
[55] M. Raczkowski, R. Frésard, A. M. Oleś, Phys. Rev. B 73, 174525 (2006).

[56] M. Raczkowski, M. Capello, D. Poilblanc, R. Frésard, A. M. Oleś, Phys. Rev. B 76, 140505(R) (2007).

[57] M. Raczkowski, R. Frésard, A. M. Oleś, Europhys. Lett. 76, 128 (2006)

[58] G. Lhoutellier, R. Frésard, A. M. Oleś, Phys. Rev. B 91, 224410 (2015)

[59] R. Frésard, W. Zimmermann, Phys. Rev. B 58, 15288 (1998).

[60] P.A. Igoshev, M.A. Timirgazin, V.F. Gilmutdinov, A.K. Arzhnikov, V.Yu. Irkhin, J. Phys: Condens. Matter 27, 446002 (2015).

[61] N. Pavlenko, T. Kopp, Phys. Rev. Lett. 97, 187001 (2006).

[62] K. Steffen, R. Frésard, T. Kopp, Phys. Rev. B 95 , 035143 (2017).

[63] R. Frésard, J. Kroha, P. Wölfle, in Theoretical Methods for Strongly Correlated Systems, edited by A. Avella, F. Mancini, Springer Series in Solid-State Sciences Vol. 171, Springer-Verlag, Berlin 2012, p. 65.

[64] T. Li, Y. S. Sun, P. Wölfle, Z. Phys. B 82, 369 (1991).

[65] R. Frésard, T. Kopp, Nucl. Phys. B 594, 769 (2001).

[66] R. Frésard, H. Ouerdane, T. Kopp, Nucl. Phys. B 785, 286 (2007)

[67] J. W. Rasul, T. Li, J. Phys. C: Solid State Phys. 21 5119 (1988)

[68] M. Lavagna, Phys. Rev. B 41, 142 (1990).

[69] T. Li, P. Bénard, Phys. Rev. B 50, 17837 (1994).

[70] Th. Jolicœur, J.C. Le Guillou, Phys. Rev. B 44, 2403 (1991).

[71] Y. Bang, C. Castellani, M. Grilli, G. Kotliar, R. Raimondi, Z. Wang, Int. J. of Mod. Phys. B 6, 531 (1992); Proceedings of the Adriatico Research Conference, Miniworkshop Strongly Correlated Electrons Systems III, eds. Yu Lu, G. Baskaran, A. E. Ruckenstein, E. Tossati, World Scientific Publishing Co., Singapore 1992.

[72] D. Vollhardt, Rev. Mod. Phys. 56, 99 (1984).

[73] D. Vollhardt, P. Wölfle, P. W. Anderson, Phys. Rev. B 35, 6703 (1987). 